\title{
Adélie penguins' extensive seasonal migration supports dynamic Marine Protected Area planning in Antarctica
}

Jean-Baptiste Thiebot ${ }^{1}$, Yan Ropert-Coudert ${ }^{2}$, Thierry Raclot ${ }^{3}$, Timothée Poupart ${ }^{2,4}$, Akiko Kato $^{2}$ and Akinori Takahashi ${ }^{1}$ ${ }^{I}$ National Institute of Polar Research, 10-3 Midori-cho, Tachikawa, Tokyo, Japan

${ }^{2}$ UMR 7372 CEBC, CNRS - La Rochelle Université, Villiers-en-Bois, France

${ }^{3}$ UMR7178 IPHC DEPE, CNRS - Université de Strasbourg, Strasbourg, France

${ }^{4}$ School of Life and Environmental Sciences, Deakin University, Burwood, Victoria, Australia

Spatiotemporal dynamics of ecosystems can challenge the pertinence of Marine Protected Area (MPA) planning. Seasonal environmental changes are extreme in polar regions, however MPA planning in East Antarctica relies mostly on species' summer distribution only. Thirteen Adélie penguins were tracked from Ile des Pétrels (Terre Adélie), and their seasonal distribution and behaviour were compared to the proposed "D’Urville Sea-Mertz" MPA. During the phase of high fooddemand preceding moult, penguins used mostly (68.4\%) this proposed area. However, following autumnal sea-ice extension, penguins migrated north-westwards: overall, $73 \%$ of their locations were outside the MPA proposal, and this was up to $>99 \%$ during winter (in July), the season when penguins maximized their dive depth and time (August and September, respectively). This study thus supports the proposal of implementing a "krill no-take zone" policy in this MPA, in line with the pre-moult foraging of these krill predators in this area. Further protection of the year-round habitats of migratory Adélie penguins could be achieved by inter-connecting the East Antarctic MPA proposals along the ice edge during winter, thereby mirroring the ecosystem's seasonal dynamics. 